\title{
A NEW ROV DESIGN: ISSUES ON LOW DRAG AND MECHANICAL SYMMETRY
}

\author{
Rui M. F. Gomes+ \\ Alexandre Sousa+ \\ Sérgio Loureiro Fraga+ \\ Alfredo Martins* \\ João Borges Sousa+ \\ Fernando Lobo Pereirat \\ +USTL - Underwater Systems and Technology Laboratory \\ Faculdade de Engenharia da Universidade do Porto \\ Rua Dr. Roberto Frias s/n \\ 4200-465 Porto, Portugal \\ \{rgomes,ajsousa,slfraga,jtasso,flp\}@fe.up.pt \\ +ISEP - Instituto Superior de Engenharia do Porto \\ Rua de S. Tomé 4200 Porto, Portugal \\ amartins@dee.isep.ipp.pt
}

\begin{abstract}
This paper reports the design of a new remotely operated underwater vehicle (ROV), which has been developed at the Underwater Systems and Technology Laboratory (USTL) - University of Porto. This design is contextualized on the KOS project (Kits for underwater operations). The main issues addressed here concern directional drag minimization, symmetry, optimized thruster positioning, stability and layout of ROV components. This design is aimed at optimizing ROV performance for a set of different operational scenarios. This is achieved through modular configurations which are optimized for each different scenario.
\end{abstract}

Keywords: Remotely operated vehicles, mechanical design, low drag and symmetry design, optimized thruster positioning.

\section{INTRODUCTION}

This paper reports the design of a new remotely operated underwater vehicle (ROV). This work has been done at the Underwater Systems and Technology Laboratory from Porto Utriversity (see [1]) in the context of the KOS project (Kit for underwater operations) and in cooperation with the Instituto de Engenharia Mecânica e Gestão Industrial (INEGI).

The Underwater Systems and Technology Laboratory (USTL) was founded in 1997 to promote research, development, deployment, and operation of advanced systems and technologies in oceanographic and environment field studies. These include: autonomous and operator assisted vehicles, which are small unmanned vehicles that are either autonomous or operated remotely by humans, respectively; and sensor networks, i.e. large sets of sensors each of which, in addition to sensing capabilities, have processing and communication capabilities. We have been operating the ISUSUS autonomous underwater vehicle (AUV) since 1997. Since then, we designed and developed: IES ROV system for the inspection of underwater structures; low cost autonomous underwater vehicle (AUV) for coastal oceanography; and sensor modules for remote environmental data collection.

The requirements for the new ROV design result from the experience acquired with the operation of the IES ROV [2] at harbours, rivers and sea (see Fig. 1 and TABLE I). A typical ROV operation is described in [3]. In these operations the ROV is subject to significant disturbances which include moderate currents, and thruster-to-environment interactions. Depending on the mission profile, it may be necessary to mount additional payload such as sensors or a robotic arm. This payload is typically heavy and cumbersome; this poses non-trivial constraints to ROV trimming.

Some mission profiles, such as the inspection of underwater structures, often require great maneuverability in surge and sway. Other mission profiles require optimized drag coefficients for robust and efficient operation under adverse environmental conditions.

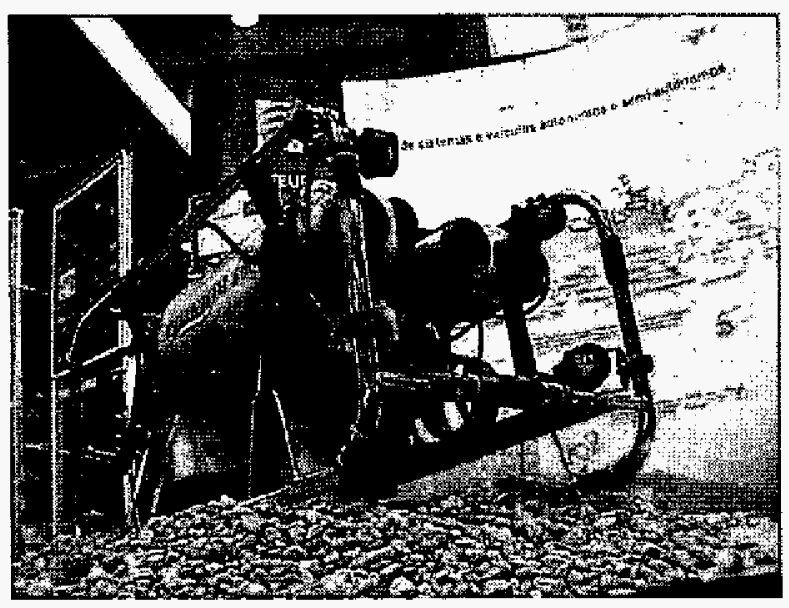

Fig. 1. IES ROV

These mission profiles require, on the one hand, a significant force system and, on other hand, optimized vehicle shapes and thruster positioning. We address both issues in the design of the new ROV. Namely we have studied: a low drag shape to face moderate aquatic currents; symmetry issues to facilitate control tasks and enhance performance; vectored thruster configurations and static regulation of dimensions to align the generated force system in the direction of the center of drag; placement of components to maximize the distance between the center of buoyancy and the center of mass for improved stability. Our design includes modular components to facilitate payload placement and ROV configuration. 
TABLE I

IES ROV TECHNICAL DATA

\begin{tabular}{|c|c|}
\hline Inspection system & Video Camera, Inspector, zoom 12:1 (ROS) \\
\hline & $\begin{array}{l}\text { Camera Pan and Tilt unit (Imenco) } \\
\text { Mechanism: } \pm 180^{\circ} \text {, } \\
\text { Lights } 600 \mathrm{~W} \text { (DSP\&L) }\end{array}$ \\
\hline Navigation sensors & $\begin{array}{l}\text { Doppler Velocity Log, Argonaut (Sontek) } \\
\text { Inertial Unit, HG1700 (Honeywell) } \\
\text { Digital Compass, TCM2 (PNI) } \\
\text { Acoustic Beacons, LBL 20-30KHz } \\
\text { Pressure sensor, (Data Instruments) }\end{array}$ \\
\hline Thrusters & $\begin{array}{l}4 \text { DC Motors, } 120 \mathrm{~V}, 1 / 8 \mathrm{HP} \text { : } \\
2 \text { thrusters on surge: } 80 \mathrm{~N} \\
1 \text { thruster on sway: } 50 \mathrm{~N} \\
1 \text { thruster on hesve: } 50 \mathrm{~N}\end{array}$ \\
\hline On-board computer & $\begin{array}{l}\text { PC-104 stack } \\
\text { QNX Real Time Operating System } \\
\text { CAN Local Bus. } \\
\text { Ethernet interface with the operator's console }\end{array}$ \\
\hline
\end{tabular}

\begin{tabular}{ll}
\hline Vehicle & Max. Working Depth: $300 \mathrm{~m}$ \\
specifications & Length: $1.20 \mathrm{~m}$ \\
& Height: $0.60 \mathrm{~m}$ \\
Width: $0.67 \mathrm{~m}$ \\
Weight: $115 \mathrm{Kg}$ \\
& \\
\hline Umbilical cable & Diameter: $11 \mathrm{~mm} 13.1 \mathrm{~mm}$ \\
& Breaking strain: $525 \mathrm{~kg} 530 \mathrm{~kg}$ \\
& Wt. in air: $100 \mathrm{~kg} / \mathrm{km} 134 \mathrm{~kg} / \mathrm{km}$ \\
& Composition: \\
& $1 \times$ Shielded Twisted Pairs for Video \\
& $1 \times$ Shielded Twisted Quad for Telemetry \\
& $2 \times$ Power conductors \\
& $1 \times$ Ground wire
\end{tabular}

The paper is organized as follows: in section II we present the main requirements for the KOS system, in section III we discuss the design solutions, and in section IV we elaborate on the mechanical design, and on drag and symmetry issues. Finally, in section $\mathrm{V}$ we provide some conclusions and discuss future work.

\section{SYSTEM REQUTREMENTS}

There are four types of mission requirements for the KOS system:

1) Inspection of underwater structures. This is the primary mission for the KOS system. The basic inspection kit includes a pan \& tilt mounted color video camera, lights, a laser image scaling system, a pencil-beam sonar, and a three degree of freedom (DOF) robotic arm. The basic inspection kit may be supplemented with additional sensors and tools to be mounted on the robotic arm. These include a corrosion meter, a marker, and a scrapper. In these missions the ROV is prone to shocks with the underwater structures and it is supposed to withstand moderate currents when operating close to the structures.

2) Underwater archeology. These mission profiles concern the inspection of archeological sites and the retrieval of light artifacts. These missions are restricted to external observations for the purpose of safety. In addition to the basic inspection kit, the ROV mounts a container for the artifacts which might have been picked up with the robotic arm.

3) Occanographic and environmental field studies. These mission specifications are quite diverse and range from video imaging of marine life to high speed data collection of environmental parameters. In these missions the ROV is required to mount oceanographic sensors which include Conductivity, Temperature, and Depth (CTD), backscatter, particle analyzer, fluorimeter, water quality sonde, etc.

4) Test-bed for advanced control and coordination concepts. This mission requirement is dictated by the research activities of the laboratory which include integrated control [4] and navigation [5] schemes, and cooperative control under limited communications. To do this, the ROV is required to mount different navigation sensors as well as acoustic modems for underwater communications.

In addition to these mission requirements the $\mathrm{KOS}$ system is constrained to:

1) Stay within the weight and size range of the IES system to facilitate operations and logistics.

2) Use the same computer, navigation, lighting, and acoustic localization sub-systems as the ones mounted on the IES subsystem to reduce development costs.

3) Share the tether, winch, and power generation sub-systems with the IES system to reduce the initial operational costs. This poses an upper bound on the power available at the surface at $2.3 \mathrm{kw}$. Later, we will be able to remove this limitation if necessary.

We derived the technical specifications for the KOS system from the mission requirements and the design constraints. These are summarized in TABLE $I$ in terms of the mechanical, payload, actuation, and power sub-systems.

TABLE II

KOS SYSTEM TECHNICAL SPECIFICATIONS

\begin{tabular}{|c|c|c|}
\hline Sub-system & Technical specifications & Justification \\
\hline Mechanical & $\begin{array}{l}\text { Symmetric shape it } x, y, z \\
\text { Passively stable roll/ pitch } \\
\text { Light materials } \\
\text { Extermal shock absorbers } \\
\text { Low drag on surge and sway } \\
\text { Spare room dry compartments } \\
\text { Shock-mounted compartments } \\
\text { Good weight/buoyancy ratios } \\
\text { for the ROV components } \\
\text { Static dissipation }\end{array}$ & $\begin{array}{l}\text { Control authority } \\
4 \text { DOF dynamics } \\
\text { Performance } \\
\text { Robustness } \\
\text { Performance } \\
\text { Configuration } \\
\text { Robustness } \\
\text { Performance } \\
\text { Power utilization }\end{array}$ \\
\hline Payload & $\begin{array}{l}\text { Multiple mounting positions } \\
\text { Multiple connectors } \\
\text { Generic payload port } \\
\text { Adjustable mounting fixtures }\end{array}$ & $\begin{array}{l}\text { Configuration } \\
\text { Configuration } \\
\text { Flexibility } \\
\text { Weight/geometry } \\
\text { distribution }\end{array}$ \\
\hline Actuation & $\begin{array}{l}\text { Thrust available at zero velocity } \\
\text { Maximum speed 2-3 knots } \\
\text { High thruster efficiency } \\
\text { Thrust/weight ratio } 0.4 \\
\text { Static adjustable mounting } \\
\text { Minimize interactions of jets } \\
\text { Forward/backward symmetry } \\
\text { Operate in currents of } 0.5 \text { ms-1 }\end{array}$ & $\begin{array}{l}\text { Performance } \\
\text { Fast motions } \\
\text { Performance } \\
\text { Performance } \\
\text { Configuration } \\
\text { Performance } \\
\text { Maneuverability } \\
\text { Performance } \\
\end{array}$ \\
\hline Power & $\begin{array}{l}\text { Reduced losses } \\
\text { High input voltage }\end{array}$ & $\begin{array}{l}\text { Power utilization } \\
\text { Power transmition }\end{array}$ \\
\hline
\end{tabular}




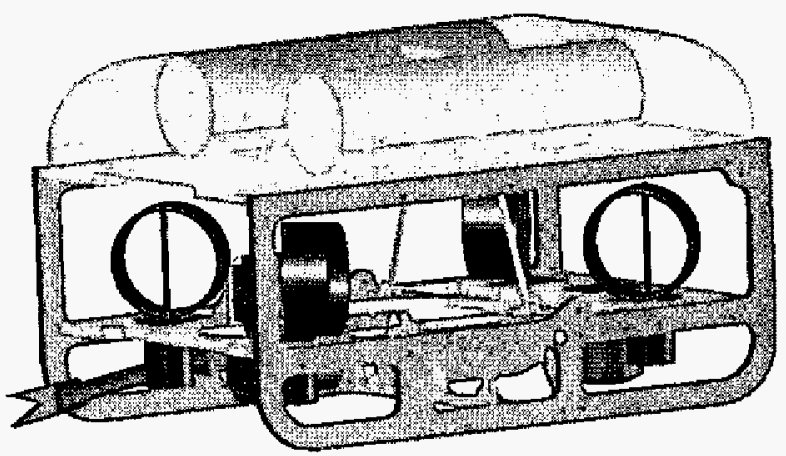

Fig. 2. 3D view of the KOS ROV

\section{SYSTEM DESIGN}

In this section we discuss the KOS ROV design concepts and solutions. The final ROV design is depicted in Fig. 2. The following section is dedicated to the issues of mechanical design with special emphasis on symmetry and drag.

\section{A. Design concepts}

The main design concepts for the KOS system were the result of a process of successive refinements, where we used the calculations described in section $\mathrm{IV}$ to analyze each concept and to further improve it. The initial design concept resembled the IES system with improved symmetry and performance. The design process is briefly described in section IV. The KOS system design concepts are described next.

The ROV is composed by three stacked horizontal sections. The upper section consists of two compartments for electronics which are mounted inside a flotation module. In addition it mounts the pencil-beam sonar, the acoustic navigation system, and a vertical thruster. The four ends of the compartments, two per compartment, include underwater connectors for payload and actuators. The middle section is basically empty, except for structural fixtures to mount the propulsion system for surge, sway, and yaw. These fixtures allow us to change the mounting position of the thrusters. This configuration minimizes jet-to-jet and jet-to-structure interactions thus maximizing thruster performance. The lower section consists basically of the payload in addition to the Doppler Velocity Log (DVL) navigation sensor, which is required to have a clear line of view in the downward direction.

The actuation system is composed by a vectored thruster system for surge, sway, and yaw. The system consists of four thrusters mounted at angles less than $45^{\circ}$ with respect to the surge axis. In these degrees of freedom the vehicle is over-actuated. We use this feature to control the force system in several modes. In the differential mode the system is capable to deliver thruster instantaneously at zero velocity. We use one thruster to the control motions in $\mathbf{z}$.

The ROV frame and the flotation compartments are made of composite materials for improved weight/buoyancy ratios. The electronic compartments are made of aluminum. The frame is made of Polyethylene plates which are machined to the specifications.

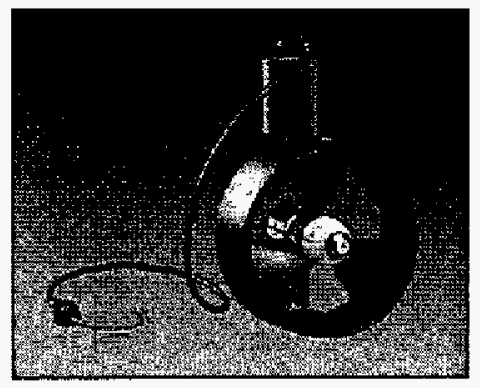

Fig. 3. Seaeye SI-MCT01 Thruster

The flotation module is made of reinforced fiberglass and is also machined to the specifications. We built several flotation modules which are easily interchangeable to adjust the hydrostatic stability of the ROV. The lateral and vertical panels are secured to one another with an L-shaped component. Four vertical struts ensure the rigidity of the ensemble.

The mechanical configuration of the ROV is designed to facilitate control design [4] namely in what concerns buoyancy, weight, and drag symmetry.

In order to accommodate different payloads and mission profiles we designed the ROV for three different mechanical configurations: base, data collection, and intervention. All the configurations share the same navigation sensors. In the base configuration the ROV mounts the pan \& tilt mounted color video camera, lights, a laser image scaling system, a pencil-beam sonar, and a two function manipulator with two fingers. In the data collection configuration, in addition to the base payload, the ROV mounts the sensors for oceanographic data collection. Alternatively it mounts an acoustic modem for underwater communications. The intervention configuration is intended for heavy duty operations with a 6 DOF manipulator, possibly with a hydraulic power pack. In this configuration, the ROV mounts a new lower section which is designed to accommodate the manipulator system. The new lower section is designed for not disrupting the weight/buoyancy relations of the base configuration.

In each configuration the user is able to optimally configure the weight, drag, and buoyancy distributions. To do this, the user adjusts the mounting positions for each thruster and for the payload, and selects an appropriate flotation compartment. This is done with the help of computational models which were also used in the design of the system.

In the reminder of the paper, and unless stated otherwise, we refer to the nominal configuration

\section{B. Actuation}

We have selected 5 Seaeye SI-MCT01 thrusters (see Fig. 3) for the actuation system: four are mounted on the middle section of the ROV in a statically adjustable vectored thrust configuration (refer to Fig. 2); one is mounted on the upper section to provide vertical thrust. The Seaeye thrusters are almost symmetric and have a high power-to-thrust ratio, providing respectively 130 and $128 \mathrm{~N}$ maximum forward and reverse thrust at $300 \mathrm{~W}$. The propeller diameter is 180 $\mathrm{mm}$. With these thrusters we achieve a maximum

\footnotetext{
${ }^{1}$ www.seaeye.com
} 
weight-to-thrust ratio in surge within the technical specifications.

Both the mounting position in the $\mathrm{z}$ direction and the orientation can be adjusted individually for each of the four thrusters. This provides for the static configuration of the force system: the total forward thrust can vary from 0 to 520 $\mathrm{N}$; and the force system can be aligned with drag force for optimized dynamic response. This feature is used to optimize the ROV for speed or for station-keeping.

In addition to the static configuration of the force system, we have introduced a thruster allocation module to optimize the real-time operation of this over-actuated system [6]. The thruster allocation module includes several modes for operation as well as the mode switching logic. There are modes for differential control, fault handling in the presence of thruster failures, minimization of power consumption, and maximization of directional forces. Differential control mode is often used in inspection scenarios. Basically the thrusters are producing opposing force systems so that resulting force system is zero. The advantage of this mode is that instantaneous torque is available in a linear fashion around zero velocity. This is because each thruster is already producing a significant thrust.

Another commonly used technique for over-actuated systems consists of using the pseudo-inverse of the non-square thrust actuation matrix $B[7]$ :

$$
B^{\prime}=B^{T}\left(B B^{T}\right)^{-1}
$$

This strategy minimizes the distribution of control energy for each degree of freedom (DOF).

\section{Components}

The following table presents the list of the mechanical characteristics of the main components of the KOS system in its base configuration.

TABLE III

$$
\text { COMPONENTS }
$$

\begin{tabular}{ll|l}
\hline Component & Dimetrsions [1 w h] or [d 1] $(\mathrm{m})$ & Weight $(\mathrm{Kg})$ \\
\hline Trusters & {$[00.090 .11] / 00.22$ Nozzle } & 21.6 \\
\hline Manipulator & {$[00.110 .55]$} & 4 \\
\hline camera & {$[00.090 .21]$} & 2.5 \\
\hline pencilbean & {$[00.080 .20]$} & 1.8 \\
\hline CTD & {$[00.060 .60]$} & 2 \\
\hline DVL & {$[00.160 .19]$} & 3.6 \\
\hline P\&T & {$[0.120 .140 .14]$} & 4.4 \\
\hline Frame & {$[1.200 .760 .20]$} & 25 \\
\hline Total (w/ extras) & & $90($ aprox) \\
\hline
\end{tabular}

\section{Power sub-system}

The power budget is given in TABLE IV.

TABLE IV POWER BUDGET

\begin{tabular}{ll}
\hline System & Power (W) \\
\hline Thrusters (5 units) & 1750 \\
\hline Lights (4 units) & 600 \\
\hline $\begin{array}{l}\text { Computational (CPu, Net, AD, Can, } \\
\text { SDLC, Rs232, rellay, Net hub) }\end{array}$ \\
\hline $\begin{array}{l}\text { Sensors (IMU, DVL, Compass, } \\
\text { Pencilbeam, Depth, Cortosion) }\end{array}$ \\
\hline Pan \& tilt, manipulator, lasers & 70 \\
\hline $\begin{array}{l}\text { Other Electronics (Voltage, } \\
\text { Temperature and Pressure monitors) }\end{array}$ & 5 \\
\hline Total & 2480 \\
\hline
\end{tabular}

The IES ROV umbilical cable specifications were a major design constraint. In fact, this power budget would require the IES ROV umbilical cable to deliver $48 \mathrm{~A} @ 48 \mathrm{~V}$; this is beyond the cable specifications. In order to reuse this cable we choose to drive the KOS ROV with nominal $230 \mathrm{~V}$ $\mathrm{AC}$ (10A max). The on-board power sub-system consists of two stages of AC-DC and DC-DC converters from Vicor ${ }^{2}$. We use two $1500 \mathrm{~W}$ VI-ARM modules in the AC-DC stage and six 500W V300A48C500A DC-DC converters in the second stage; each rectifier module connects to three DC-DC converters; the six $48 \mathrm{~V}$ outputs are mounted in parallel to deliver close to $3000 \mathrm{~W}$ at the $48 \mathrm{~V}$ power supply bus. This is only possible because the Vicor DC-DC converters have a load sharing feature.

\section{ISSUES IN MECHANICAL DESIGN}

In this section we discuss the issues leading to mechanical structure described in the previous section and to the placement of the ROV components in this structure. This was done with several objectives in mind: facilitating control design (mechanical and control co-design), optimizing both static and dynamic behavior, and maximizing functional utilization.

Here we discuss the issues concerning static and dynamic behavior, and mechanical and control co-design. These include roll/pitch passive stability, thruster positioning, and drag, weight and buoyancy symmetries and reduction. We do this with reference to the equations of motion for an underwater vehicle [7] [11]. These are presented next, in the body-fixed frame coordinates:

$$
\begin{gathered}
\mathrm{M} \dot{\mathrm{v}}+\mathrm{C}(\mathrm{v}) \mathrm{v}+\mathrm{D}(\mathrm{v}) \mathrm{v}+\mathrm{g}(\eta)=\tau \\
\dot{\eta}=\mathrm{J}(\eta) \mathrm{v}
\end{gathered}
$$

where $v$ is the velocity of the vehicle in the body-fixed frame, $\eta$ the position and the orientation in the inertial frame, $M$ the inertia and added mass matrix, $C(v)$ the Coriolis and centripetal matrix, $D(v)$ the damping matrix, $g(\eta)$ the restoring forces and moments and $\tau$ the body-fixed forces from the actuators [8] and disturbances, such as currents and waves. Equation (1.3) converts body-fixed velocities to inertial velocities. The weight and buoyancy forces are called the restoring forces. These forces are responsible for bringing the vehicle back to its stable position in roll and pitch, which are defined to be zero in that position. The equations for the restoring moments on roll and pitch are given by the following equations:

$$
\begin{gathered}
\mathrm{K}_{\phi}=-\mathrm{BG}_{\mathrm{y}} \mathrm{W} \cos \theta \cos \phi+\mathrm{BG}_{\mathrm{z}} \mathrm{W} \cos \theta \sin \phi \\
\mathrm{M}_{\theta}=\mathrm{BG}_{\mathrm{z}} \mathrm{W} \sin \theta+\mathrm{BG}_{\mathrm{x}} \mathrm{W} \cos \theta \cos \phi
\end{gathered}
$$

where $\mathrm{W}$ is the gravity force and $B \mathrm{G}_{\mathrm{i}}$ the distance between the center of gravity and the center of buoyancy in the $x, y$ and $\mathrm{z}$ directions, i.e., $\left(\mathrm{x}_{\mathrm{G}}-\mathrm{x}_{\mathrm{B}}\right),\left(\mathrm{y}_{\mathrm{G}}-\mathrm{y}_{\mathrm{B}}\right),\left(\mathrm{z}_{\mathrm{G}}-\mathrm{z}_{\mathrm{B}}\right)$. The conditions for passive roll and pitch stability are easily derived from these equations. The restoring forces should be zero for zero angles in pitch and roll, and should drive the vehicle back to zero pitch and roll away from these angles. The first condition requires both $\mathrm{BG}_{x}$ and $\mathrm{BG}_{y}$ to be zero. The second condition requires $\mathrm{BG}_{\mathrm{z}}$, which is called the metacentric height, to be different from 0 . In fact, the restoring moments are proportional to $\mathrm{BG}_{z}$ for given pitch and roll angles.

\footnotetext{
${ }^{2}$ www.vicor.com
} 


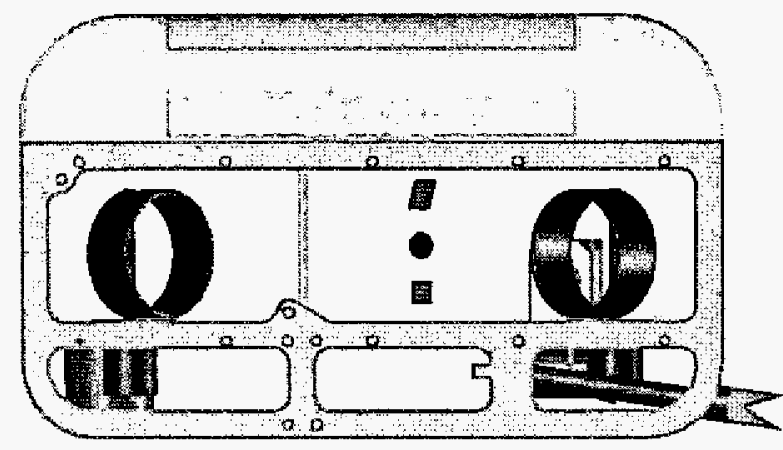

Fig. 4. Lateral view of KOS ROV. The buoyancy center is marked with red parallelogram; the drag center is marked with blue circle and gravity center with a green square.

The $\mathrm{D}(\mathrm{v})$ term in equation (1.2) models effects like potential damping, wave drift damping, skin friction and vortex shedding damping. The main contribution comes from quadratic linear friction also known as drag. The main equation for drag is given next

$$
F_{D}=-\frac{1}{2} \rho C_{D}\left(R_{n}\right) A|u| u .
$$

Here $u$ is the vehicle velocity in one of the main directions, $A$ the area of the component, $\rho$ the water density and $C_{D}$ the drag coefficient. The drag coefficient itself is also a function of the Reynolds number which is given by the following equation

$$
\mathrm{R}_{n}=\frac{U D}{v}
$$

where $U$ is the body velocity, $D$ is the characteristic length (i.e. the diameter in case of a cylinder) and $v$ the water viscosity.

Basicatly, the mechanical design problem consists of determining optimal configurations for $M, D(v), g(\eta)$, and for the actuation part of the force system $\tau$ under the design constraints summarized in TABLE II. This is basically a multi-criteria optimization problem which we solved iteratively. First we came up with a hydrostatic design conceming mainly $M, C(v)$, and $g(\eta)$. Afterwards we improved the drag, the main component of $D(v)$, and the actuation components of the force system. This also imposed modifications to the previous design. The final solution was reached after a few iterations.

We developed Matlab ${ }^{3}$ programs to calculate and optimize the hydrostatic and drag parameters. We also plan to use these programs to trim and balance the ROV before operations.

The developed Matlab program to calculate the hydrostatic parameters determines the centers of gravity and buoyancy, the metacentric height, and the entries for the added mass matrix. The computations take as inputs the mass and dimensions for each component of the ROV. Each component is characterized by its own gravity and buoyancy centers, under averaging assumptions. A sensitivity analysis may be performed to check if the averaging assumption is not valid in some components. If this is this case, we run more detailed calculations.

\footnotetext{
${ }^{3}$ www.mathworks.com
}

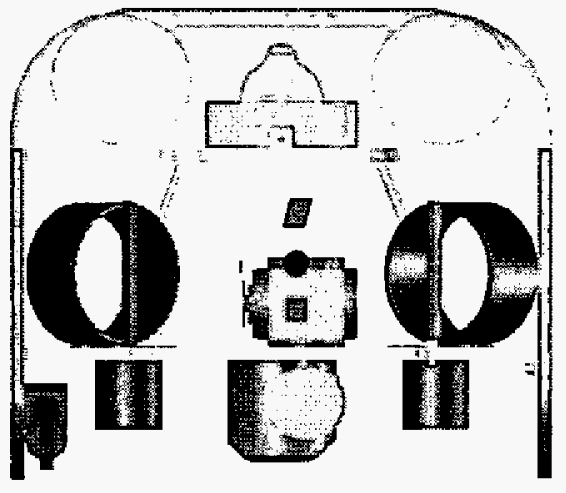

Fig. 5. Front view of KOS ROV. The buoyancy center is marked with red parallelogram; the drag center is marked with blue circle and gravity center with a green square.

In our initial design concept the ROV resembled the IES ROV: a buoyancy module plus thrusters on top and two cylindrical comportments for electronic compartments below. After some calculations we found out that the buoyancy produced by those compartments far exceeded their weight and would lead to an unstable design. This is why we have moved these compartments to the upper section of the ROV. This enabled us to move the 4 heavy thrusters to the middle section of the vehicle with a significant improvement in the restoring moment. The pan\&tilt mounted video camera and the robotic arm are required to be installed in the bottom front part of the ROV for inspection purposes. This led us to the 3 sections design concept, which was also used for constructive purposes.

The payload in the bottom section is particularly heavy and is not evenly distributed. This required the addition of extra flotation and weight for balancing. We used the Matlab program to calculate how.

Fig. 4 and Fig. 5 display the locations for both the centers of gravity and buoyancy in the final design for the ROV base configuration. These are vertically aligned. The $\mathrm{BG}_{\mathrm{z}}$ is approximately $10 \mathrm{~cm}$ which leads to good passive roll and pitch stability.

Next we address the problem of drag minimization and force alignment. The problem of mechanical and control co-design is of interest to us at this point: we would like both the actuation of the vehicle to be aligned with the drag force, at least for pure longitudinal, lateral and vertical motions, and the drag force to be minimized. This alignment ensures that, for constant velocities, the vehicle will not endure any moment on roll, pitch and yaw, thus facilitating control design and improving control performance. To achieve both goals we have to determine optimal configurations for the $D$ (v) matrix and for the structure of the actuation part of the force system $\tau$. We do this for pure longitudinal, lateral and vertical motions only; this requires the calculation of the centers of drag in the $x y, x z$, and yz planes; it is much more difficult to compute drag for coupled motions.

We have developed a Matlab program to calculate the $x y$, $x z$, and $y z$ centers of drag. To do this the ROV is decomposed into a set of basic components in each of these planes. Each component is characterized by an area and a drag coefficient. The area is the total area exposed to the water while the drag coefficient is obtained from the tables and graphs in [9] [10] [12]. For example, for a cylindrical body with a diameter of $0.08 \mathrm{~m}$ and moving at a velocity of 
$\mathrm{Im} / \mathrm{s}$ over the water, the Reynolds number is 80000 , if we assume the water viscosity to be $10^{-6} \mathrm{~m}^{2} / \mathrm{s}$, and the drag coefficient that we obtain from the tables is 1.0 . With the area and the drag coefficient we calculate the drag force. Doing this for all the components in a given plane we are able to determine the drag force for each pure motion and for a particular velocity over the water. Again we are considering averaging assumptions. Notice that under accelerations different from zero this model is not valid. Fig. 4 and Fig. 5 show the locations of the corresponding centers of drag. With the drag force application point calculated, we can design the thruster application point to be approximately coincident with it in order to have stability with constant speed. The vehicles behavior during the accelerations will not be predictable because the proposed approach is based only on constant speeds.

We also went through a few iterations to find the optimal positioning for the ROV components. Again we did this with the belp of the Matlab program.

Finally, we checked and validated our drag calculations with the COSMOSFloWorks ${ }^{4}$ CFD program. In addition to validating our calculations, this program enabled us to visualize the fluid flows and the distributions of pressure which provided for a better understanding of the underlying hydrodynamic behavior as we can infer from picture Fig. 6 .

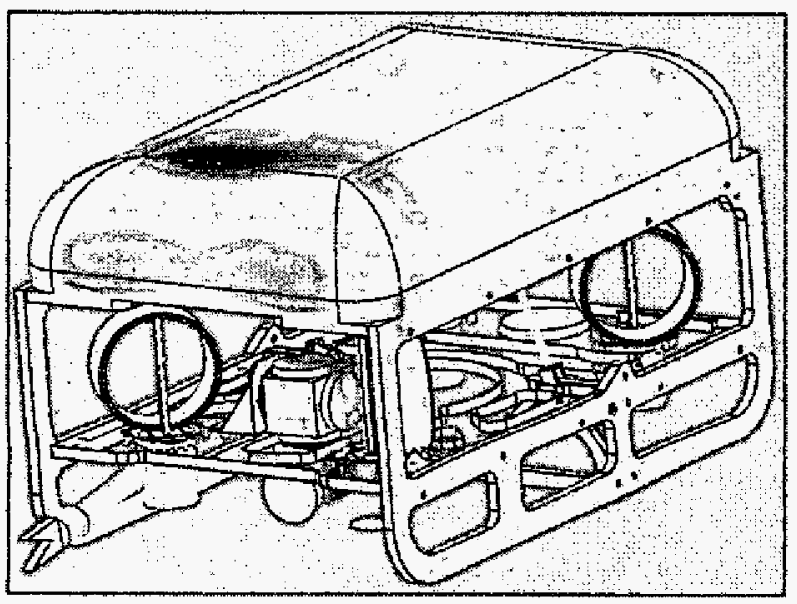

Fig. 6. Pressure distribution on the vehicle

\section{CONCLUSIONS AND FUTURE WORK}

In this paper we have described the specification and the design concepts for a new ROV, and discussed the mechanical design, namely in what concerns modularity, configurations, and mechanical and control co-design. The initial tests for the new ROV are scheduled for May 2005.

There are several directions for future work: i) to further develop our design tools to account for parametric uncertainty, in the context of robust optimization, and multi-criteria optimization; ii) to use these tools in conjunction with system identification procedures to further refine the performance of our vehicles; and iii) to extend our design analysis to coupled motions. Finally, we are interested in developing an integrated toolset for design optimization.

\footnotetext{
${ }^{4} \mathrm{http}: / / \mathrm{www} \cdot \cos \operatorname{mosm} . \operatorname{com} / \cos$ mosfloworks.htm
}

\section{ACKNOWLEDGMENTS}

The KOS project is funded by Agência de Inovação. Rui Gomes and Sérgio Fraga are funded by Fundação para a Ciência e Tecnologia (FCT). The authors would like to thank the valuable comments from Prof. Anthony Healey. We also want to thank Hugo Ferreira with his help on graphics.

\section{REFERENCES}

[1] Underwater Systems and Technology Laboratory, $<$ http://www.fe.up.pt/sts/>

[2] Matos, A., Cruz, N., Martins, A., and Lobo Pereira, F., " Development and Implementation of a Low-Cost LBL Navigation System for an AUV ", Oceans'99 MTS/IEEE, Article 2, Session 2E, Seattle, WA, 1999.

[3] Rui M. F. Gomes, João B. Sousa and Fernando Lobo Pereira, "Integrated maneuver and control design for ROV operations", OCEANS 2003, San Diego, CA, USA, September 22-26, 2003.

[4] Rui Gomes, J. Borges Sousa and Lobo Pereira, "Modelling and control of the IES project ROV", Proceedings of the European Control Conference, Cambridge, UK, 2003.

[5] Luís Madureira, Márcio Correia e Anfbal Matos, "Sistema Integrado De Navegaçăo Para Um Vé́culo Submarino"

[6] W. Webtser and J. Borges de Sousa, "OptimumAllocation for Multiple Thrusters", Proceedings of the ISOPE'99, Brest, France, June 1999.

[7] Thor I. Fossen. Guidance and Control of Ocean Vehicles. John Whiley and Sons, 1995.

[8] D. Yoerger L. Whitcomb. Development, comparison, and preliminary experimental validation of nonlinear dynamic thruster models. IEEE Journal of Oceanic Engineering, Vol. 24, 1999.

[9] Sighard F. Hoerver, "Fluid Dynamic Drag", Published by author, second edition, 1965

[10] E. Lewis. Principles of Naval Architecture. Society of NavalArchitects and Marine Engineers, 1989.

[11] A. J. Healey and D. Lienard, "Multivariable sliding mode control for autonomous diving and steering of unmanned underwater vehicles," IEEE J. Oceanic Eng., vol. 18, July 1993.

[12] J. N. Newman. Marine Hydrodynamics. MIT Ptess, 1977. 